\title{
Farewell to GBM-O: Genomic and transcriptomic profiling of glioblastoma with oligodendroglioma component reveals distinct molecular subgroups
}

\author{
Benjamin H. Hinrichs ${ }^{1 \dagger}$, Scott Newman ${ }^{2 \dagger}$, Christina L. Appin ${ }^{3}$, William Dunn ${ }^{4}$, Lee Cooper ${ }^{4}$, Rini Pauly,
} Jeanne Kowalski ${ }^{2,6}$, Michael R. Rossi ${ }^{1,7}$ and Daniel J. Brat ${ }^{1,4^{*}}$

\begin{abstract}
Introduction: Glioblastoma with oligodendroglioma component (GBM-O) was recognized as a histologic pattern of glioblastoma (GBM) by the World Health Organization (WHO) in 2007 and is distinguished by the presence of oligodendroglioma-like differentiation. To better understand the genetic underpinnings of this morphologic entity, we performed a genome-wide, integrated copy number, mutational and transcriptomic analysis of eight (seven primary, one secondary) cases.

Results: Three GBM-O samples had IDH1 (p.R132H) mutations; two of these also demonstrated 1p/19q co-deletion and had a proneural transcriptional profile, a molecular signature characteristic of oligodendroglioma. The additional IDH1 mutant tumor lacked 1p/19q co-deletion, harbored a TP53 mutation, and overall, demonstrated features most consistent with IDH mutant (secondary) GBM. Finally, five tumors were IDH wild-type (IDHwt) and had chromosome seven gains, chromosome 10 losses, and homozygous 9p deletions (CDKN2A), alterations typical of IDHwt (primary) GBM. IDHwt GBM-Os also demonstrated EGFR and PDGFRA amplifications, which correlated with classical and proneural expression subtypes, respectively.

Conclusions: Our findings demonstrate that GBM-O is composed of three discrete molecular subgroups with characteristic mutations, copy number alterations and gene expression patterns. Despite displaying areas that morphologically resemble oligodendroglioma, the current results indicate that morphologically defined GBM-O does not correspond to a particular genetic signature, but rather represents a collection of genetically dissimilar entities. Ancillary testing, especially for IDH and 1p/19q, should be used for determining these molecular subtypes.
\end{abstract}

Keywords: Glioblastoma, Glioblastoma with oligodendroglioma component, IDH mutation, 1p/19q co-deletion

\section{Introduction}

Glioblastoma (GBM) is the highest grade infiltrating astrocytoma and the World Health Organization (WHO) recognizes several morphologic patterns. Glioblastoma with Oligodendroglioma component (GBM-O), WHO grade IV, was recently described as a diffusely infiltrative,

\footnotetext{
* Correspondence: dbrat@emory.edu

${ }^{\dagger}$ Equal contributors

'Department of Pathology and Laboratory Medicine, Emory University School of Medicine, 1648 Pierce Dr NE, Atlanta, GA 30307, USA

${ }^{4}$ Department of Biomedical Informatics, Emory University, 36 Eagle Row 5th

Floor South, Atlanta, GA 30322, USA

Full list of author information is available at the end of the article
}

high-grade astrocytic neoplasm displaying necrosis, and also demonstrating discrete areas of oligodendroglial differentiation [1]. Some studies have suggested that oligodendroglioma-like areas in GBM may confer a favorable prognosis, yet this remains controversial [2-8]. Moreover, it is unclear whether GBM-O represents a distinct entity with defining molecular alterations and clinical behavior or a collection of genomically disparate tumors containing similar morphologic elements $[2-6,8,9]$.

Prior studies suggested that GBM-O is enriched for $I D H$ mutations and has fewer PTEN deletions than other forms of GBM [2]. Co-deletion of chromosomes 1p and 

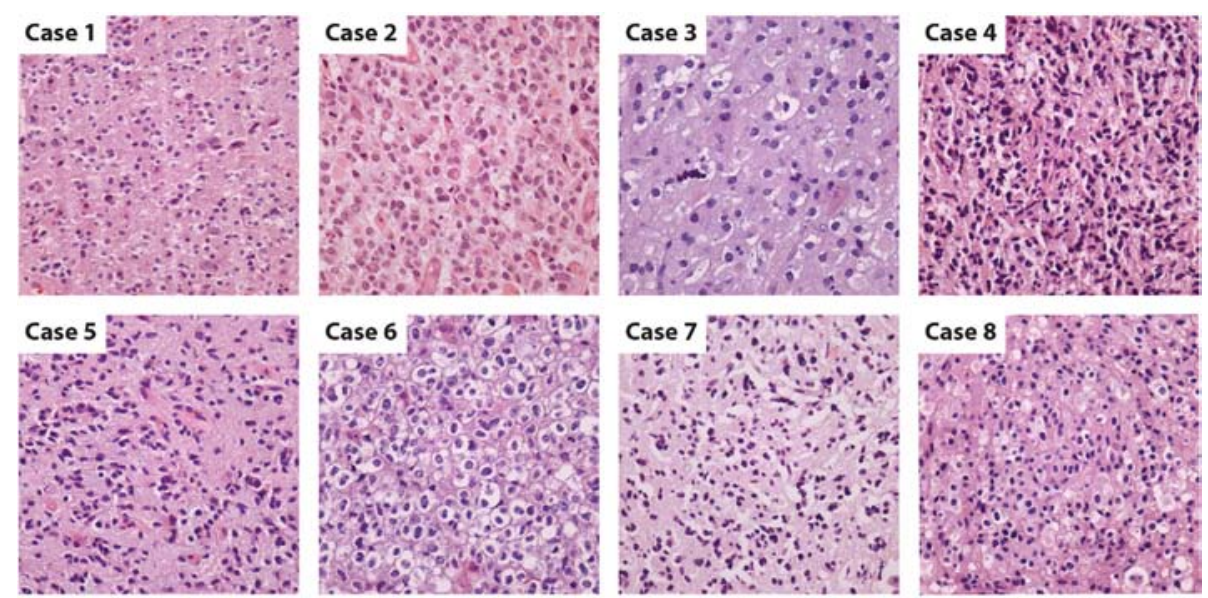

Fig. 1 Photomicrographs of GBM-O cases. Images show representative areas of macrodissected tumor (H\&E, 20X). In all cases, submitted tissue included regions of oligodendroglial differentiation

$19 \mathrm{q}$ is the molecular signature of oligodendroglioma and associated with enhanced therapeutic response and longer survival [10], yet most studies of GBM-O have identified $1 \mathrm{p} / 19 \mathrm{q}$ co-deletion only in a modest subset $[6,8]$. Conversely, EGFR amplifications, which are mutually exclusive from $I D H$ mutations and $1 \mathrm{p} / 19 \mathrm{q}$ co-deletions, have been documented in GBM-Os and may define a clinically relevant subset [3]. Advanced whole genome molecular platforms can provide additional biologic insights into tumor subtypes, above that offered by morphology or a limited biomarker panel [4, 11, 12]. Therefore, in order to better characterize GBM-O, we performed copy number microarray, whole transcriptome RNA-sequencing and gene panel deep sequencing.

\section{Materials and methods \\ Patient information and histopathology}

All tumor tissue was obtained from patients who underwent surgical resection and pathological evaluation at Emory University Hospitals from 2007 through 2011. Two neuropathologists reviewed all hematoxylin and eosin (H\&E)-stained slides from each case concurrently using a multi-headed microscope. GBM-O was the original pathologic diagnosis in all cases, and all diagnoses were rendered using World Health Organization 2007 criteria [1]. Paraffin embedded tissue sections of 10micron thickness were macro-dissected to maximize viable tumor submitted for genomic analysis. Six of eight samples contained $>95 \%$ viable tumor, while a single case each contained significant necrosis or normal tissue (25\%, case $8 ; 40 \%$, case 5 , respectively). In all cases, submitted tissue included regions of oligodendroglial differentiation (Fig. 1). Summary clinical data and molecular/cytogenetic testing results are shown in Fig. 2a. FISH testing $(E G F R, 1 \mathrm{p} / 19 \mathrm{q})$ and mutant IDH1 expression by immunohistochemistry, were previously performed as described [2]. Immunohistochemistry for ATRX (1:100; Sigma-Aldrich Corp., St. Louis, MO, USA) assessed nuclear expression in select cases.

\section{Nucleic acid isolation and QC}

Serial sections were evaluated for tumor proportion and necrosis prior to nucleic acid extraction. DNA and RNA were extracted using the QIAGEN (Valencia, CA) AllPrep FFPE kit. Genomic DNA was quantitated using the

a

\begin{tabular}{|c|c|c|c|c|c|c|c|}
\hline Case & Diagnosis & Gender & Diagnosis Age & Survival & FISH EGFR & FISH 1p/19q & $\mathrm{IHCIDH}-1$ \\
\hline 1 & GBM-0, Grade IV* & $M$ & $29 \mathrm{yrs}$ & $41 \mathrm{mo}$ & Negative & Co-deleted & Positive \\
\hline 2 & GBM-O, Grade IV & M & $68 \mathrm{yrs}$ & $20 \mathrm{mo}$ & Negative & Co-deleted & Positive \\
\hline 3 & GBM-O, Grade IV & $\mathrm{F}$ & $51 \mathrm{yrs}$ & $69 \mathrm{mo}$ & Negative & Negative & Negative \\
\hline 4 & GBM-O, Grade IV & M & $78 \mathrm{yrs}$ & $8 \mathrm{mo}$ & Amplified & Negative & Negative \\
\hline 5 & GBM-O, Grade IV & M & $57 \mathrm{yrs}$ & $16 \mathrm{mo}$ & Amplified & 1p deletion & $n$ Negative \\
\hline 6 & GBM-O, Grade IV & M & $73 \mathrm{yrs}$ & $2 \mathrm{mo}$ & Amplified & Negative & Negative \\
\hline 7 & GBM-O, Grade IV & $\mathrm{F}$ & $37 \mathrm{yrs}$ & $19 \mathrm{mo}$ & Amplified & Negative & Negative \\
\hline 8 & GBM-O, Grade IV & M & $47 \mathrm{yrs}$ & $7 \mathrm{mo}$ & Amplified & Negative & Negative \\
\hline
\end{tabular}

b

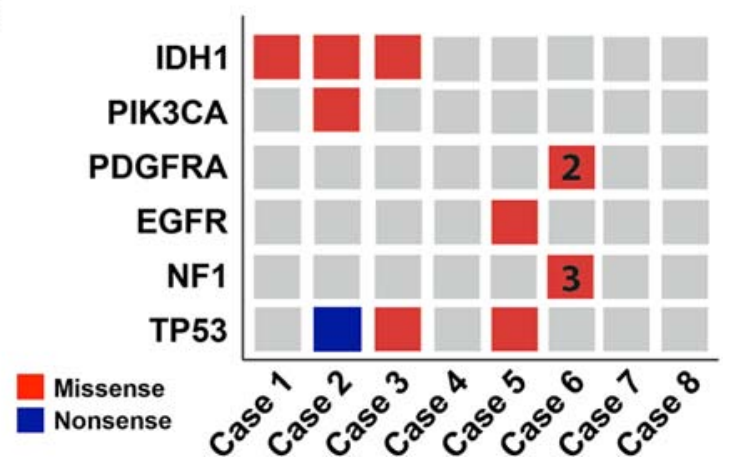

Fig. 2 Clinicopathologic features of GBM-O cases and results of targeted Fluidigm DNA sequencing. a Case details including previously obtained FISH (EGFR, 1p/19q) and IDH1 R132H immunohistochemistry results. *prior diagnosis was fibrillary astrocytoma, grade II b Nonsynonymous mutations were detected in 5/8 cases (12 missense, 1 nonsense). Numerals in boxes indicate number of mutations if more than one 
Qubit dsDNA high sensitivity (HS) kit (ThermoFisher Scientific, Waltham, MA); total RNA was quantitated and quality checked using the Agilent 2100 Bioanalyzer with the RNA 6000 pico kit (Agilent Technologies, Santa Clara, CA).

\section{Targeted amplicon DNA sequencing}

A custom amplicon-library preparation assay was developed for the Fluidigm Access Array (Fluidigm Corporation, South San Francisco, CA) using the D3 Assay Design software. Coding exons of 12 genes previously reported as recurrently mutated in GBM were targeted (BRAF, EGFR, IDH1, IDH2, NF1, NRAS, PDGFRA, PIK3CA, PIK3R1, PTEN, RB1, TP53) [13] (Additional file 1: Table S1). Briefly, the Bioinformatic variant/indel (collectively referred to as "variants") pipeline comprised trimming low quality bases and adapters using Trimmomatic 0.32 [14]; merging overlapping paired end reads with ea-utils fastq-join version 1.1.2-686 (https://code.google.com/p/ea-utils/), and keeping the highest quality base call from the overlapping region; alignment to the hg19 human reference genome using the BWA-mem aligner version $0.7 .5 \mathrm{a}$ [15]; calling potential variants with VarScan2 requiring at least $20 \times$ coverage, five nonreference reads with a minimum base quality score of 20 and a minimum variant allele frequency of 0.25 [16]. Predicted variants were annotated with ANNOVAR [17] (Additional file 2: Table S2). Since we used archival FFPE tissue without matched normal DNA, we carried out extensive filtering of predicted variants and manually verified variants using Integrative Genomics Viewer [18]. Variants not present in the Catalogue of Somatic Mutations in Cancer (COSMIC) database were required to have $1,000 \times$ coverage $[19,20]$. Likely germline variants that were observed at greater than $1 \%$ frequency in either the Thousand Genomes Project or Exome Variant Server were filtered out $[21,22]$. Those found in dbSNP without previously being linked to cancer were also removed [23].

\section{Copy number microarray}

Extraction and hybridization of genomic DNA to Illumina HumanCytoSNP-12v2.1-FFPE SNP arrays was according to manufacturer's instructions. Data were processed and analyzed with BioDiscovery Nexus software (Hawthorne, CA) using SNPRank segmentation and a segmented $\log 2$ ratio of 0.09 to call gains, 0.3 for amplifications, -0.135 for losses and -0.45 for homozygous deletions. Combined segmented data was viewed using Copy Number Explorer software [24].

\section{Whole transcriptome sequencing}

RNAseq library preparation and Illumina HiSeq sequencing were carried out by Beckman Coulter Genomics
(Danvers, MA) using Illumina TruSeq RNA library preparation and HiSeq2500 sequencing. RNA sequencing produced approximately 50 million reads per sample. We aligned paired-end fastq files to the hg19 human reference genome using Tophat 2.0.6 under standard parameters [25]. RefSeq Transcripts were quantified and transformed into FPKM values using Cufflinks 2.02 [26].

\section{Gene expression-based molecular classification}

We used TCGA GBM samples to derive an RNASeq gene list with which to classify our GBM-O samples. 164 TCGA samples had been classified into one of four subgroups based on expression microarray profiles and also had accompanying RNA-Seq data [13]. We initially performed non-negative matrix factorization consensus clustering with a k-value of four using gene pattern software [27] and the NMF module. We omitted samples whose NMF cluster and microarray expression subclass were discordant, leaving 115 samples. We used shrunken centroids, deployed through the pamr R-package to identify the gene sets that best classified each subtype [28]. The best-performing classification sets were a subset of the original 840-gene classifier described by Verhaak et al. [29] and comprised of 129 genes specific to classical, 160 messenchymal, 65 neural and 131 proneural genes (Additional file 3: Table S3). We used Gene Set Variation Analysis (GSVA) [30], an enhanced version of Single Sample Gene Set Enrichment Analysis (ssGSEA) [31], to classify expression microarray profiles from TCGA patient samples. GSVA classified $94 \%$ of samples correctly (as assessed by cluster membership based on GSVA score) (not shown). We deployed GSVA through the GSVA Rpackage [30], to our GBM-O samples.

\section{Results}

\section{Clinicopathologic features of GBM-O}

From January, 2008 through December, 2011, we made the diagnosis of GBM-O 28 times. Eight of these cases were chosen based on availability of tissue and diversity of clinical diagnostic markers. Of these, seven were primary and one secondary (case 1), the latter having been diagnosed as a fibrillary astrocytoma, grade II four years prior. Ages at diagnosis ranged from 29- to 78-years (mean, 55years) and clinical follow-up revealed patient survivals ranged from 2- to 69-months (mean, 23 months; median, 17.5 months). Tumors were classified as GBM-O if a discrete area of oligodendroglial differentiation (occupying at least a single $100 \times$ microscopic field) occurred in the setting of a high-grade astrocytic neoplasm with necrosis [2]. Oligodendroglial differentiation was recognized as glioma cells containing round, regular nuclei with only mild variation accompanied by a delicate capillary vascular network and occasionally by cytoplasmic clearing [32]. Alternatively, high-grade astrocytic differentiation was defined 
as glioma cells with elongated, hyperchromatic nuclei and irregular nuclear contours [32]. Photomicrographs in Fig. 1 show representative areas of tumor analyzed for each case.

Previous diagnostic testing included FISH analysis of EGFR and $1 \mathrm{p}$ and $19 \mathrm{q}$ as well as immunohistochemistry for IDH1 p.R132H (Fig. 2a). Cases harboring different combinations of genetic alterations were chosen to capture the molecular diversity displayed in GBM-O. Two were positive for mutant IDH1 protein and had 1p/19q co-deletions and were negative for EGFR amplification. Four cases were EGFR-amplified and did not show mutant IDH1 protein expression or 1p/19q co-deletion; an additional case was EGFR-amplified, IDH1 wild-type and had a $1 \mathrm{p}$ deletion. The remaining case did not show any molecular alteration detected by FISH or IHC staining. Of note, the presence of $1 \mathrm{p} / 19 \mathrm{q}$ co-deletion did not correlate with higher percentages of oligodendroglioma component.

\section{Detection of coding variants using targeted Fluidigm DNA sequencing}

We performed DNA sequencing to identify coding variants in 12 genes recurrently mutated in GBM (BRAF, EGFR, IDH1, IDH2, NF1, NRAS, PDGFRA, PIK3CA, PIK3R1, PTEN, RB1, TP53) [13] (Fig. 2b, Additional file 2: Table S2). In agreement with IHC results, cases 1 and 2 displayed IDH1 (c.395G > A; p.R132H) mutations. Case 2, which was $1 \mathrm{p} / 19 \mathrm{q}$ co-deleted, also contained a PIK3CA mutation, concordant with previous studies showing correlation among these alterations [11]. Additionally, case 3 also had an p.R132H mutation, despite being reported as negative by R132H IHC [33, 34], as well as a TP53 mutation (c.481G > A, p.A161T) that has been previously reported in diffuse gliomas (COSM10739). The cooccurrence of TP53 and IDH mutations is seen in the large majority of lower-grade (WHO grades II-III) diffuse astrocytomas, as well as secondary GBMs (WHO grade IV) and defines a lineage of $I D H$ mutated tumors distinct from $1 \mathrm{p} / 19 \mathrm{q}$ co-deleted oligodendrogliomas [11, 34]. Although $A T R X$ alterations are frequently seen together with $I D H$ and TP53 mutations, we did not demonstrate loss of ATRX expression by IHC in this case [35, 36].

The remaining five cases were all $I D H$ wild-type (IDHwt). EGFR and PDGFRA mutations were noted exclusively in $I D H$ wt GBM-Os and all NF1 mutations were also noted in this subset. EGFR and PDGFRA mutations were both seen in the context of EGFR and PDGFRA amplification respectively (see below), an association previously observed in primary GBM [29].

\section{Copy number microarray}

Detection of specific somatically-acquired copy-number aberrations (CNAs) aids in CNS tumor classification, and may provide important insight into tumor biology [29]. To determine if GBM-O displayed distinct patterns of CNA, we performed copy number microarray analysis. While FISH is used diagnostically to assess CNAs at defined loci (EGFR, PTEN and 1p/19q) microarray testing can detect copy number changes genome wide. A full list of copy number segments is in Additional file 4: Table S4.

There was general agreement between microarray and diagnostic FISH testing (Fig. 3): Cases 1 and 2 showed 1p/19q deletion by both FISH and microarray. Case 3 was negative by FISH and microarray for $1 \mathrm{p} / 19 \mathrm{q}$ deletion and EGFR amplification. Cases 4, 5, 7 and 8 were EGFR amplified by both FISH and microarray. Case 6 was EGFR amplified by FISH, but the reported percentage of cells showing amplification $(28 / 200,14 \%)$ was below our detection threshold of approximately $30 \%$ for this microarray. Case 5 demonstrated $1 \mathrm{p}$ deletion and 19q gain by FISH. Microarray showed a gain of chromosome 19, but no loss of $1 \mathrm{p}$. Reanalysis with a more sensitive segmentation algorithm, ASCAT [37, 38] (data not shown) identified a terminal $55 \mathrm{Mb}$ deletion of $1 \mathrm{p}$. These results are consistent and rule out a whole arm loss of chromosome 1.

Gains, losses and amplifications of other loci were evident but there was no single CNA found across all cases; rather, three general patterns were apparent: One group (cases 1 and 2) had 1p/19q co-deletion, and lacked gains of chromosome 7, amplifications of EGFR or losses of chromosome 10. Another group (cases 4, 5, 6, 7, and 8) demonstrated either broad gains of chromosome 7 or focal amplifications of 7p11.2 including EGFR, and most showed loss of chromosome 10 (including PTEN) as well as heterozygous deletion of $9 \mathrm{p}$ with or without focal homozygous deletion of $C D K N 2 A$. Several of these cases also had other CNAs previously described in GBM but not oligodendroglioma including PDGFRA amplification (cases 5 and 6) and MDM2 amplification (case 4) [13]. Lastly, case 3 displayed a third pattern of copy number alteration. While showing alterations overlapping those seen in the latter group (gain of chromosome 7 and heterozygous deletion of $9 \mathrm{p}$ ), case 3 also demonstrated alterations not present in other cases including loss of chromosome 3 and $9 q$, as well as gains of $16 p$ and chromosomes 5 and 21 .

\section{Gene expression-based molecular classification}

Previous gene expression studies identified four GBM expression subtypes - proneural, classical, neural and mesenchymal - named after the expression signature's resemblance to transcriptional profiles of neural cell types [29]. We next determined if our GBM-O samples were all classified as the same expression subgroup or, alternatively, represented a combination of two or more subgroups. We performed RNASeq gene expression profiling and classified these data using Gene Set Variation 


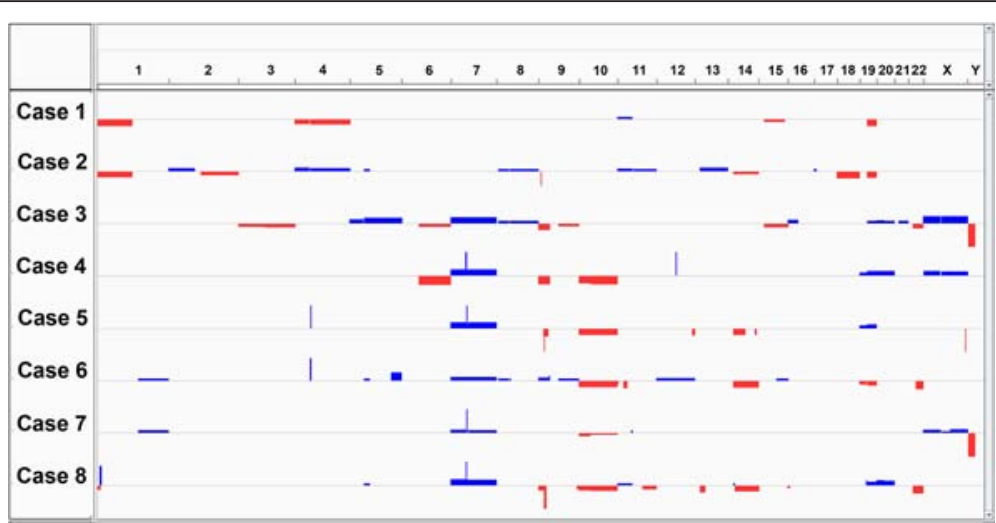

Fig. 3 Patterns of recurrent copy-number aberrations (CNAs) identified using Illumina microarray. Focal amplifications of 7p11.2 (EGFR) and gains of chr7 were present in 4/8 and 6/8 cases, respectively, and were mutually exclusive of 1p/19q co-deletion and IDH1 R132H mutation (cases 1 and 2). Loss of chr10 (PTEN) and homozygous 9p21.3 loss (CDKN2A) occurred in 4/4 and 2/4 cases with EGFR amplification, respectively. Focal gains involving $4 \mathrm{q} 12$ (PDGFRA) were present in 2 cases

Analysis (GSVA). GSVA expression enrichment scores for GBM-O cases are displayed in Fig. 4. A column Zscore of $>0.5$ was used as a cutoff to determine whether a tumor sample was enriched for a particular subtype expression signature. Additional file 5: Figure S1 displays nominal scores indicating correlation between sample transcriptional profile and expression subtype compared across samples. Expression values for select genes are provided in Additional file 6: Table S5.

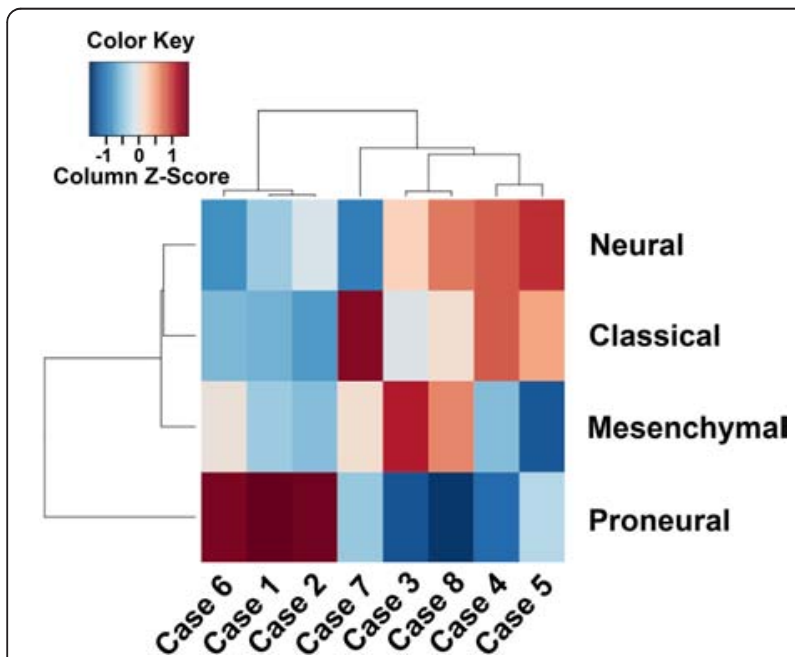

Fig. 4 Gene expression profile heat map. A positive enrichment score (column Z-score) indicates the tumor sample expression profile and the genes in that particular gene set are positively correlated, whereas a negative enrichment score indicates the opposite. Five tumors demonstrated expression signatures enriched for one subtype, while the remaining three had transcriptional profiles enriched for two. Three tumors showed exclusive classification as the proneural subtype (cases 1, 2, and 6), and one tumor each was classified exclusively as classical and mesenchymal (cases 7 and 3, respectively). Cases 4 and 5 showed both classical and neural signatures, while case 8 showed both mesenchymal and neural subtype expression patterns
Overall, all four GBM expression subtypes were represented among the eight GBM-Os (Fig. 4). Five demonstrated expression signatures strongly enriched for one subtype, while the remaining three had transcriptional profiles enriched for two subtypes (Fig. 4 and Additional file 5: Figure S1). Three tumors showed exclusive classification as the proneural subtype (cases 1 , 2 , and 6), and one tumor each was classified exclusively as classical and mesenchymal (cases 7 and 3, respectively). Cases 4 and 5 showed enrichment for both classical and neural genes, while case 8 showed both mesenchymal and neural signatures.

The three tumor samples classified as proneural were tightly clustered and included two of three IDH1 mutant tumor samples, consistent with other studies demonstrating this strong association [3, 29]. Also in concordance with previous studies, all cases demonstrating proneural gene expression signatures showed increased expression levels of the proneural gene $D C X$ [29]. An additional GBM-O with proneural expression profile exhibited high-level PDGFRA amplification and gene expression, also typical of this transcriptional subtype [29].

Cases with classical signatures demonstrated substantially elevated EGFR expression, a discriminating feature of this subtype [29]. Consistent with previous reports, tumor samples in the classical subgroup also demonstrated increased expression of $L F N G$, a gene encoding for an $\mathrm{O}$-fucosylpeptide 3-beta- $\mathrm{N}$-acetylglucosaminyltransferase known to affect Notch signaling [29].

Two cases showed enrichment for the mesenchymal gene set and both demonstrated increased expression of CHI3L1, a marker consistently elevated in this subtype [39]. RELB and TNFRSF1A, genes involved in the NF-kB and tumor necrosis factor super family pathways, were also elevated, consistent with prior reports [29]. One of the GBM-Os classified as mesenchymal (case 8) demonstrated 
a significant percentage of necrosis (25\%), a feature observed in mesenchymal GBMs in the TCGA cohort [29].

Three GBM-O samples demonstrated enrichment for neural gene sets and two of the three showed substantially elevated expression of the neuron marker SYT1 in concordance with previous studies [29]. Although two normal brain tissue samples analyzed by Verhaak et al. [29] were categorized as the neural subtype, only one of the three current tumor samples contained a significant percentage of benign tissue (case 5).

\section{Integration of molecular platforms}

Previous studies of GBM have shown associations between transcriptional signatures, somatic mutations and DNA copy number alterations [3, 29]. To investigate whether these associations are also present in GBM-O, we correlated patterns of somatic mutation and DNA copy number alterations with expression subtype (Fig. 5). Overall, the selected GBM-Os could be divided into three groups. The first (cases 1 and 2) consisted of GBM-Os with IDH1 mutations and $1 \mathrm{p} / 19 \mathrm{q}$ co-deletions and lacked DNA copy number alterations typical of GBM such as EGFR amplification, gains of chromosome 7 and losses of 10 [13]. IDH1 mutations and 1p/19q codeletion have been shown to be strongly correlated in GBM-O [2, 40]. Case 2 also contained a PIK3CA mutation, a finding previously documented with $I D H$ mutations and $1 \mathrm{p} / 19 \mathrm{q}$ co-deletion [11]. Both IDH1 mutant, $1 \mathrm{p} / 19 \mathrm{q}$ co-deleted tumors showed a proneural gene

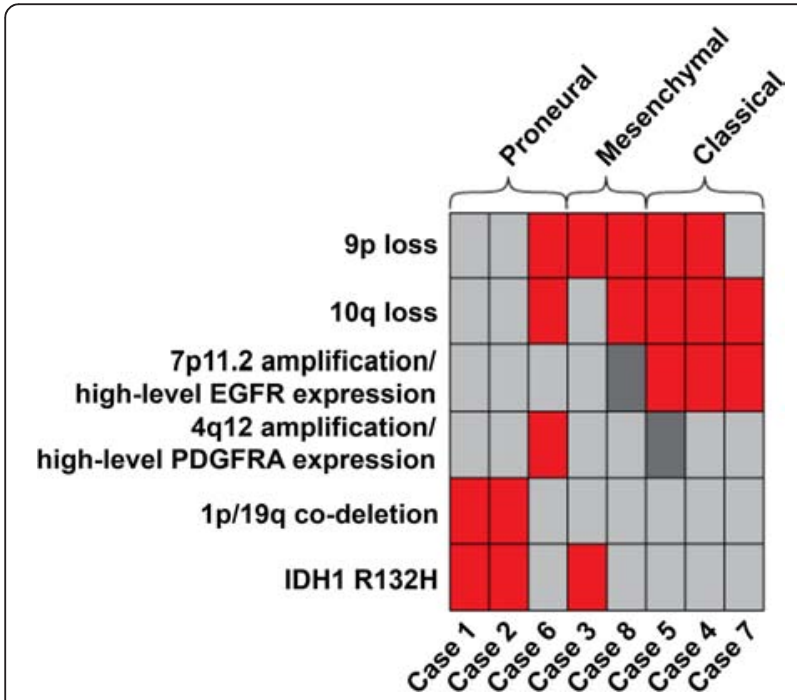

Fig. 5 Overview of molecular aberrations in GBM-O cases. Each column represents a single case and cases are grouped by expression signature subtype. In regards to EGFR and PDGFRA alterations, red boxes indicate samples demonstrated both DNA amplification and high-level gene expression, whereas samples with dark grey boxes showed only amplification. Light grey boxes indicate negative for mutation or balanced chromosomal status expression signature, and, therefore, displayed genetic alterations resembling those seen in oligodendrogliomas [34].

An additional tumor (case 3) was IDH1 mutated, but lacked 1p/19q co-deletion and harbored a TP53 mutation. This genetic pattern is typical of $I D H$ mutant diffuse atrocytomas of WHO grades II, III and IV [41]. While this case showed additional characteristics of astrocytic lineage, including gain of chromosome 7 and heterozygous deletion of $9 \mathrm{p}$, it lacked typical alterations of primary GBM such as EGFR amplification, chromosome 10 loss and homozygous deletion of CDKN2A. Taken together, these findings are most consistent with an IDH mutant GBM, often referred to clinically as "secondary" GBM.

The remaining 5 GBM-Os were $I D H$ wild-type (IDHwt). Four of the IDHwt tumors were amplified for EGFR (cases 4, 5, 7 and 8), had broad gains of chromosome 7, loss of chromosome 10, CDKN2A deletion (two cases) and showed enrichment for the classical expression gene sets (three cases). Among cases with classical transcription signatures, high-level EGFR amplification corresponded to significantly elevated EGFR expression. The GBM-O that harbored an EGFR mutation also showed EGFR amplification and had a classical expression signature.

One of the IDHwt tumors (case 6) demonstrated a proneural expression signature and exhibited the characteristic constellation of $4 \mathrm{q} 12$ focal amplification involving PDGFRA, high levels of PDGFRA gene expression, as well as PDGFRA mutation. This case also demonstrated chromosome 7 gain and losses of 10 and 9p, indicating additional copy number similarity with primary GBM.

Among IDHwt tumors, transcriptional profiles were predominantly classified as classical and neural; however, this subgroup also contained representatives from the proneural and mesenchymal subtypes. IDHwt GBM-Os in the current study, therefore, displayed gene expression diversity similar to GBMs in the TCGA cohort [29]. Coupled with the classic DNA copy-number alterations discussed above, the IDHwt subgroup overall demonstrated molecular alterations most consistent with primary GBM.

\section{Conclusions}

Glioblastoma with Oligodendroglioma component (GBMO) was introduced by the WHO classification in 2007 as one of the morphologic patterns of GBM [1]. Clinical outcomes data and biomarker profiles have, however, yielded inconsistent results and the clinical significance and biology of GBM-O is not well understood. While morphology has informed CNS tumor classification for decades, molecular testing can also define distinct genetic entities with specific clinical outcomes. Therefore, we employed copy 
number microarray, whole transcriptome RNASequencing and gene panel deep sequencing to better characterize the GBM-O entity. Our results showed that despite having overlapping morphologic features, our GBM-O cohort was composed of three discrete molecular subgroups, each with characteristic coding variants, CNAs and gene expression patterns.

Previous investigations have also attempted to clarify the significance of GBM-O through molecular testing. The presence of $1 \mathrm{p} / 19 \mathrm{q}$ co-deletions has received particular attention given its association with oligodendrogliomas and improved clinical outcomes [41]; however, reported frequencies having ranged from 4 to $30 \%[2-6,8]$. Compared with conventional GBM, GBM-Os show consistently increased frequencies of IDH1 mutations, though enrichment of GBM-O cohorts with secondary GBMs may explain this finding $[2,3,8]$. Despite the evidence for an increased association between GBM-O and IDH mutations and $1 \mathrm{p} / 19 \mathrm{q}$ co-deletions, there is variation in clinical outcome studies with some showing a favorable prognosis when compared to conventional GBMs [2, 6-8, 42], while others do not [3-5]. Lastly, investigations show wideranging frequencies of EGFR amplification (39-71\%) as well as LOH of 10q (0-58 \%) highlighting the molecular heterogeneity seen in GBM-O [2, 3, 6, 42]. Our current data indicates that the variations in clinical outcomes and biomarker status reported in these investigations may be due to the inclusion of multiple molecular genetic entities with overlapping morphologic features into the analyses. While additional molecular variation could potentially exist in the setting of morphologic diversity, previous data has indicated genomic stability across morphologic phenotypes [5].

A crucial subdivision of diffuse gliomas is based on $I D H$ mutational status, since IDH mutant tumors progress more slowly and are associated with prolonged survival, grade for grade [11, 34]. The large majority of grades II and III diffuse gliomas, including oligodendrogliomas and astrocytomas, are $I D H$ mutant [11]. Only a small minority of GBMs are IDH mutant and many of these have progressed from grade II and III astrocytomas (secondary GBMs) [13]. In our analysis, three of eight GBM-Os were $I D H$ mutant, whereas five were IDHwt. Two of the $I D H$ mutant tumors in our cohort were $1 \mathrm{p} / 19 \mathrm{q}$ co-deleted recapitulating the strong positive correlation between these alterations seen in other studies of GBM-O [2, 9, 40]. These tumors also had a proneural transcriptional profile, and therefore, overall demonstrated a molecular signature that is classic for oligodendroglioma. Both patients with this molecular signature had survivals (20 and 41 months) longer than the median for this cohort (17.5 months). Integrating the genetic findings of these high grade gliomas with $I D H$ mutations and $1 \mathrm{p} / 19 \mathrm{q}$ co-deletions, as suggested by a recent international consensus of neuropathologists, would lead to their categorization as anaplastic oligodendroglioma, WHO grade III, with $I D H$ mutation and 1p/19q co-deletion, rather than GBM-O [43].

An additional tumor (case 3) was IDH1 mutated, but it lacked 1p/19q co-deletion and harbored a TP53 mutation. The constellation of IDH1 and TP53 mutation without $1 \mathrm{p} / 19 \mathrm{q}$ co-deletion is seen in diffuse astrocytomas of WHO grade II, III and IV $[11,41]$. Interestingly, the occurrence of a TP53 mutation in the context of an intact $1 \mathrm{p}$ arm as in this case, is consistent with the mutual exclusivity of $1 \mathrm{p}$ deletion and p53 overexpression observed in other GBM-O cohorts [2, 9]. Typical alterations seen in IDHwt (primary) GBM such as EGFR amplification, chromosome 10 loss and homozygous deletion of $C D K N 2 A$ were not present, although the observed gain of chromosome 7 and heterozygous deletion of 9p provide further evidence of the tumor's astrocytic lineage. Overall, the identified IDH1 and TP53 mutations, the lack of molecular alterations seen in primary GBM and the longest survival of the study indicate this case is an $I D H$ mutant form of GBM, often referred to as "secondary GBM".

$I D H$ wild-type GBM-Os in our cohort, on the other hand, were characterized by chromosome 7 gains, chromosome 10 losses, and 9p (CDKN2A) deletions; signatures of an astrocytoma lineage and most typical of IDHwt GBM $[29,41]$. This subgroup contained additional alterations characteristic of IDHwt GBM such as EGFR amplification and mutation, as well as NF1 mutations. EGFR amplified tumors were mostly associated with the classical transcriptional class, as previously described [29]. IDHwt GBM-Os also contained representatives from the proneural, mesenchymal, and neural subtypes and, therefore, display diversity homologous to GBMs in the TCGA cohort [29]. In addition, we also identified an IDHwt GBM-O with high level PDGFRA amplification and a gene expression profile aligned with the proneural class, an association also previously established in primary GBM [29]. Lastly, consistent with behavior of tumors molecularly resembling primary GBM, all IDHwt cases had survivals shorter than the mean. Average patient survival of this group, however, did not differ significantly from $I D H$ mutant tumors in this small cohort.

Recent investigations of the diffuse gliomas have indicated that $I D H$ mutations and $1 \mathrm{p} / 19 \mathrm{q}$ co-deletion provide a framework for defining molecular classes that distinguish lineages and clinical behavior [11, 41]. Diffuse gliomas with $I D H$ mutations and $1 \mathrm{p} / 19 \mathrm{q}$ codeletion correspond to oligodendrogliomas, while $I D H$ mutant tumors lacking the co-deletion typically have TP53 mutations, ATRX alterations and are considered astrocytic in differentiation [11, 34, 41]. Diffuse gliomas that are $I D H w t$ are typically high grade and have clinical behavior and genetic alterations similar of primary GBM 
[11, 13, 41]. Lastly, grade II and III diffuse gliomas that contain morphologic features of both oligodendroglioma and astrocytoma, i.e. oligoastrocytoma, have now been shown to have either the molecular signature of oligodendroglioma or astrocytoma [44]. Thus, some have questioned the existence of oligoastrocytoma at the molecular level $[44,45]$. Combining the above molecularbased approach with morphologic appearance has led to the use of an "integrated" diagnostic framework. Applied specifically to tumors diagnosed as GBM-O, recent studies have shown this diagnostic algorithm separates GBM-O into prognostically relevant groups, namely anaplastic oligodendroglioma and GBM, with the latter further classified based on IDH mutation status [36, 46]. The overall patterns of genetic alterations that we have noted in GBM-O suggest a similar conclusion and indicate that these tumors can be divided into three discrete molecular classes: 1) those that are $I D H$ mutant and $1 \mathrm{p} /$ $19 \mathrm{q}$ co-deleted have the molecular signature of a high grade oligodendroglioma; 2) those that are IDH mutant and not co-deleted have features of secondary GBM; and 3 ) those that are $I D H w t$ have the molecular signature of primary GBMs.

Therefore, despite displaying areas that morphologically resemble oligodendroglioma, the current findings indicate that these foci do not specifically signify the presence of $1 \mathrm{p} / 19 \mathrm{q}$ co-deletion, and overall lack specificity for a particular genetic signature. Taken together, the findings suggest molecular testing should be relied on for establishing diagnoses of diffuse gliomas.

\section{Availability of data and materials}

The datasets supporting the conclusions of this article are included within the article and its additional files.

\section{Additional files}

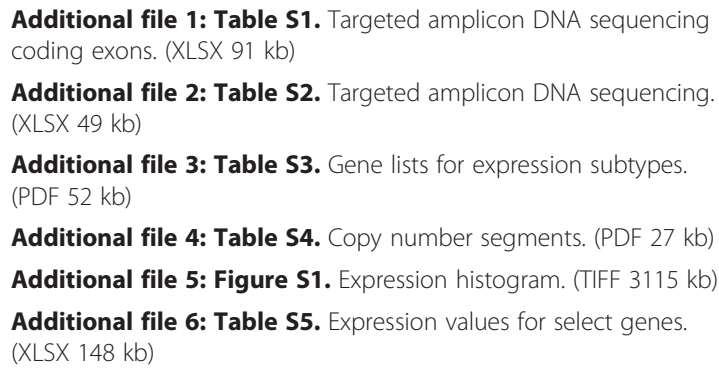

Additional file 3: Table S3. Gene lists for expression subtypes. (PDF $52 \mathrm{~kb}$ )

Additional file 4: Table S4. Copy number segments. (PDF $27 \mathrm{~kb}$ ) Additional file 5: Figure S1. Expression histogram. (TIFF $3115 \mathrm{~kb}$ ) Additional file 6: Table S5. Expression values for select genes. (XLSX $148 \mathrm{~kb}$ )

\section{Competing interests}

The authors declare that they have no competing interests.

\section{Authors' contributions}

$\mathrm{BH}$ reviewed GBM-O cases, analyzed copy number microarray data, manually verified targeted amplicon DNA variant data, and drafted the manuscript. SN processed and analyzed copy number microarray data, performed RNAseq alignment, conducted the gene expression-based molecular classification, and aided both in the development of the targeted amplicon DNA variant calling pipeline as well as in the preparation of the manuscript. CA reviewed GBM-O cases, and aided in compilation of GBM-O cases. WD and LC developed the custom amplicon-library preparation assay for the Fluidigm Access Array as well as the targeted amplicon DNA variant calling pipeline. RP and JK performed the gene expression-based molecular classification. MR performed nucleic acid isolation and QC, hybridization of genomic DNA to Illumina microarrays, RNAseq library preparation and Illumina HiSeq sequencing, and analyzed copynumber microarray data. DB reviewed GBM-O cases, aided in manuscript preparation, and was integral to the overall study design and analysis. All authors read and approved the final manuscript.

\section{Acknowledgements}

Research reported in this publication was supported in part by the Biostatistics and Bioinformatics Shared resource of Winship Cancer Institute of Emory University and NIH/NCI under award number P30CA138292. The Winship Cancer Tissue and Pathology Shared Resource, including Jennifer Shelton and Dianne Alexis, were instrumental in their assistance with this work. The work was also supported by NIH/NCI award R01 CA176659 (DJB)

\section{Author details}

${ }^{1}$ Department of Pathology and Laboratory Medicine, Emory University Schoo of Medicine, 1648 Pierce Dr NE, Atlanta, GA 30307, USA. ${ }^{2}$ Biostatistics and Bioinformatics Shared Resource, Winship Cancer Institute of Emory University, 1365 Clifton Rd, Atlanta, GA 30322, USA. ㄹDepartment of Pathology, Northwestern University of Feinberg School of Medicine, 303 East Chicago Avenue Ward 3-140W127, Chicago, IL 60611, USA. ${ }^{4}$ Department of Biomedical Informatics, Emory University, 36 Eagle Row 5th Floor South, Atlanta, GA 30322, USA. ${ }^{5}$ Greenwood Genetics Center, JC Self Research Institute, 106 Gregor Mendel Circle, Greenwood, SC 29646, USA. ${ }^{6}$ Department of Biostatistics and Bioinformatics, Rollins School of Public Health, Emory University, 1518 Clifton Road, Atlanta, GA 30322, USA. ${ }^{7}$ Department of Radiation Oncology, Emory University School of Medicine, 1648 Pierce Dr NE, Atlanta, GA 30307, USA.

Received: 24 December 2015 Accepted: 24 December 2015 औसे

References

1. Louis DN, Ohgaki H, Wiestler OD, Cavenee WK, Burger PC, Jouvet A, et al. The 2007 WHO classification of tumours of the central nervous system. Acta Neuropathol. 2007;114:97-109. doi:10.1007/s00401-007-0243-4.

2. Appin CL, Gao J, Chisolm C, Torian M, Alexis D, Vincentelli C, et al. Glioblastoma with oligodendroglioma component (GBM-O): molecular genetic and clinical characteristics. Brain Pathol. 2013;23:454-61. doi:10. 1111/bpa.12018

3. Hegi ME, Janzer RC, Lambiv WL, Gorlia T, Kouwenhoven MC, Hartmann C, et al. Presence of an oligodendroglioma-like component in newly diagnosed glioblastoma identifies a pathogenetically heterogeneous subgroup and lacks prognostic value: central pathology review of the EORTC 26981/NCIC CE.3 trial. Acta Neuropathol. 2012;123:841-52. doi:10.1007/s00401-011-0938-4.

4. Nakamura H, Makino K, Kuratsu J. Molecular and clinical analysis of glioblastoma with an oligodendroglial component (GBMO). Brain Tumor Pathol. 2011:28:185-90. doi:10.1007/s10014-011-0039-z.

5. Pinto LW, Araujo MB, Vettore AL, Wernersbach L, Leite AC, Chimelli LM, et al. Glioblastomas: correlation between oligodendroglial components, genetic abnormalities, and prognosis. Virchows Arch. 2008;452:481-90 doi:10.1007/s00428-007-0562-9.

6. Salvati M, Formichella Al, D'Elia A, Brogna C, Frati A, Giangaspero F, et al, Cerebral glioblastoma with oligodendrogliomal component: analysis of 36 cases. J Neurooncol. 2009:94:129-34. doi:10.1007/s11060-009-9815-6.

7. Vordermark D, Ruprecht K, Rieckmann P, Roggendorf W, Vince GH, Warmuth-Metz M, et al. Glioblastoma multiforme with oligodendroglial component (GBMO): favorable outcome after post-operative radiotherapy and chemotherapy with nimustine (ACNU) and teniposide (VM26). BMC Cancer. 2006;6:247. doi:10.1186/1471-2407-6-247.

8. Wang Y, Li S, Chen L, You G, Bao Z, Yan W, et al. Glioblastoma with an oligodendroglioma component: distinct clinical behavior, genetic alterations, and outcome. Neuro Oncol. 2012;14:518-25. doi:10.1093/ neuonc/nor232. 
9. Kraus JA, Lamszus K, Glesmann N, Beck M, Wolter M, Sabel M, et al. Molecular genetic alterations in glioblastomas with oligodendroglial component. Acta Neuropathol. 2001;101:311-20.

10. Smith JS, Perry A, Borell TJ, Lee HK, O'Fallon J, Hosek SM, et al. Alterations of chromosome arms $1 p$ and $19 q$ as predictors of survival in oligodendrogliomas, astrocytomas, and mixed oligoastrocytomas. J Clin Oncol. 2000;18:636-45.

11. Cancer Genome Atlas Research N, Brat DJ, Verhaak RG, Aldape KD, Yung WK, Salama SR, et al. Comprehensive, integrative genomic analysis of diffuse lowergrade gliomas. N Engl J Med. 2015;372:2481-98. doi:10.1056/NEJMoa1402121.

12. Klink B, Schlingelhof B, Klink M, Stout-Weider K, Patt S, Schrock E. Glioblastomas with oligodendroglial component-common origin of the different histological parts and genetic subclassification. Cell Oncol (Dordr) 2011;34:261-75. doi:10.1007/s13402-011-0034-8.

13. Brennan CW, Verhaak RG, McKenna A, Campos B, Noushmehr H, Salama SR, et al. The somatic genomic landscape of glioblastoma. Cell. 2013;155:462-77. doi:10.1016/j.cell.2013.09.034.

14. Bolger AM, Lohse M, Usadel B. Trimmomatic: a flexible trimmer for Illumina sequence data. Bioinformatics. 2014;30:2114-20. doi:10.1093/bioinformatics/ btu170.

15. Li H, Durbin R. Fast and accurate short read alignment with BurrowsWheeler transform. Bioinformatics. 2009;25:1754-60. doi:10.1093/ bioinformatics/btp324.

16. Koboldt DC, Zhang Q, Larson DE, Shen D, McLellan MD, Lin L, et al. VarScan 2: somatic mutation and copy number alteration discovery in cancer by exome sequencing. Genome Res. 2012;22:568-76. doi:10.1101/gr.129684.111.

17. Wang K, Li M, Hakonarson H. ANNOVAR: functional annotation of genetic variants from high-throughput sequencing data. Nucleic Acids Res. 2010;38: e164. doi:10.1093/nar/gkq603.

18. Robinson JT, Thorvaldsdottir H, Winckler W, Guttman M, Lander ES, Getz G, et al. Integrative genomics viewer. Nat Biotechnol. 2011;29:24-6. doi:10. 1038/nbt.1754.

19. Catalogue Of Somatic Mutations In Cancer (COSMIC). http://cancer.sanger. ac.uk/cosmic. Accessed Feb 2015

20. Forbes SA, Beare D, Gunasekaran P, Leung K, Bindal N, Boutselakis H, et al. COSMIC: exploring the world's knowledge of somatic mutations in human cancer. Nucleic Acids Res. 2015;43:D805-811. doi:10.1093/nar/gku1075.

21. Exome Variant Server. http://evs.gs.washington.edu/EVS/. Accessed Oct 2014

22. Genomes Project C, Abecasis GR, Auton A, Brooks LD, DePristo MA, Durbin $\mathrm{RM}$, et al. An integrated map of genetic variation from 1,092 human genomes. Nature. 2012;491:56-65. doi:10.1038/nature11632.

23. Database of Single Nucleotide Polymorphisms; dbSNP (Build 141). http://www.ncbi.nlm.nih.gov/SNP/. Accessed Nov 2014

24. Newman S. Interactive analysis of large cancer copy number studies with copy number explorer. Bioinformatics. 2015;31:2874-6. doi:10.1093/ bioinformatics/btv298.

25. Trapnell C, Pachter L, Salzberg SL. TopHat: discovering splice junctions with RNA-Seq. Bioinformatics. 2009;25:1105-11. doi:10.1093/bioinformatics/btp120.

26. Trapnell C, Roberts A, Goff L, Pertea G, Kim D, Kelley DR, et al. Differential gene and transcript expression analysis of RNA-seq experiments with TopHat and Cufflinks. Nat Protoc. 2012;7:562-78. doi:10.1038/nprot.2012.016.

27. Reich M, Liefeld T, Gould J, Lerner J, Tamayo P, Mesirov JP. GenePattern 2.0. Nat Genet. 2006:38:500-1. doi:10.1038/ng0506-500.

28. Hastie T, Tibshirani R, Balasubramanian N, Chu G. pamr: Pam: prediction analysis for microarrays. R package version 1.54.1. 2013. https://cran.r-project. org/package=pamr. Dec 2014

29. Verhaak RG, Hoadley KA, Purdom E, Wang V, Qi Y, Wilkerson MD, et al. Integrated genomic analysis identifies clinically relevant subtypes of glioblastoma characterized by abnormalities in PDGFRA, IDH1, EGFR, and NF1. Cancer Cell. 2010;17:98-110. doi:10.1016/j.ccr.2009.12.020.

30. Hanzelmann S, Castelo R, Guinney J. GSVA: gene set variation analysis for microarray and RNA-seq data. BMC Bioinformatics. 2013;14:7. doi:10.1186/ 1471-2105-14-7.

31. Barbie DA, Tamayo P, Boehm JS, Kim SY, Moody SE, Dunn IF, et al. Systematic RNA interference reveals that oncogenic KRAS-driven cancers require TBK1. Nature. 2009:462:108-12. doi:10.1038/nature08460.

32. Brat DJ, Prayson RA, Ryken TC, Olson JJ. Diagnosis of malignant glioma: role of neuropathology. J Neurooncol. 2008;89:287-311. doi:10.1007/s1 1060-008-9618-1.

33. Clark K, Voronovich Z, Horbinski C. How molecular testing can help (and hurt) in the workup of gliomas. Am J Clin Pathol. 2013;139:275-88. doi:10.1309/AJCPFO8IIDNBIJ8Y.
34. Wakimoto H, Tanaka S, Curry WT, Loebel F, Zhao D, Tateishi K, et al. Targetable signaling pathway mutations are associated with malignant phenotype in IDH-mutant gliomas. Clin Cancer Res. 2014;20:2898-909. doi:10.1158/1078-0432.CCR-13-3052.

35. Liu XY, Gerges N, Korshunov A, Sabha N, Khuong-Quang DA, Fontebasso AM, et al. Frequent ATRX mutations and loss of expression in adult diffuse astrocytic tumors carrying IDH1/IDH2 and TP53 mutations. Acta Neuropathol. 2012;124:615-25. doi:10.1007/s00401-012-1031-3.

36. Reuss DE, Sahm F, Schrimpf D, Wiestler B, Capper D, Koelsche C, et al. ATRX and IDH1-R132H immunohistochemistry with subsequent copy number analysis and IDH sequencing as a basis for an "integrated" diagnostic approach for adult astrocytoma, oligodendroglioma and glioblastoma. Acta Neuropathol. 2015;129:133-46. doi:10.1007/s00401-014-1370-3.

37. Allele-Specific Copy number Analysis of Tumors (ASCAT). http://www.crick. ac.uk/peter-van-loo/software/ASCAT. Accessed May 2015

38. Van Loo P, Nordgard SH, Lingjaerde OC, Russnes HG, Rye IH, Sun W, et al. Allele-specific copy number analysis of tumors. Proc Natl Acad Sci U S A. 2010;107:16910-5. doi:10.1073/pnas.1009843107.

39. Phillips HS, Kharbanda S, Chen R, Forrest WF, Soriano RH, Wu TD, et al. Molecular subclasses of high-grade glioma predict prognosis, delineate a pattern of disease progression, and resemble stages in neurogenesis. Cancer Cell. 2006:9:157-73. doi:10.1016/j.ccr.2006.02.019.

40. Ohgaki $H$, Kleihues P. Genetic profile of astrocytic and oligodendroglial gliomas. Brain Tumor Pathol. 2011;28:177-83. doi:10.1007/s10014-011-0029-1.

41. Riemenschneider MJ, Jeuken JW, Wesseling P, Reifenberger G. Molecular diagnostics of gliomas: state of the art. Acta Neuropathol. 2010;120:567-84. doi:10.1007/s00401-010-0736-4.

42. Hilton DA, Penney M, Pobereskin L, Sanders H, Love S. Histological indicators of prognosis in glioblastomas: retinoblastoma protein expression and oligodendroglial differentiation indicate improved survival. Histopathology. 2004;44:555-60. doi:10.1111/j.1365-2559.2004.01887.x.

43. Louis DN, Perry A, Burger P, Ellison DW, Reifenberger G, von Deimling A, et al. International Society Of Neuropathology-Haarlem consensus guidelines for nervous system tumor classification and grading. Brain Pathol. 2014;24: 429-35. doi:10.1111/bpa.12171.

44. Sahm F, Reuss D, Koelsche C, Capper D, Schittenhelm J, Heim S, et al. Farewell to oligoastrocytoma: in situ molecular genetics favor classification as either oligodendroglioma or astrocytoma. Acta Neuropathol. 2014;128: 551-9. doi:10.1007/s00401-014-1326-7.

45. Wiestler B, Capper D, Sill M, Jones DT, Hovestadt V, Sturm D, et al. Integrated DNA methylation and copy-number profiling identify three clinically and biologically relevant groups of anaplastic glioma. Acta Neuropathol. 2014;128:561-71. doi:10.1007/s00401-014-1315-x.

46. Figarella-Branger D, Mokhtari K, Colin C, Uro-Coste E, Jouvet A, Dehais $C$, et al. Prognostic relevance of histomolecular classification of diffuse adult high-grade gliomas with necrosis. Brain Pathol. 2015;25:418-28. doi:10.1111/bpa.12227.

\section{Submit your next manuscript to BioMed Central and we will help you at every step:}

- We accept pre-submission inquiries

- Our selector tool helps you to find the most relevant journal

- We provide round the clock customer support

- Convenient online submission

- Thorough peer review

- Inclusion in PubMed and all major indexing services

- Maximum visibility for your research

Submit your manuscript at www.biomedcentral.com/submit 